\title{
Asinius Pollio und Augustus: Geschichtsschreibung im frühen Principat
}

\author{
Krešimir Matijević
}

Mysterium Augustus. Wohl kaum ein antiker Herrscher wird von der modernen Forschung noch 2000 Jahre nach seinem Tod so unterschiedlich eingeschätzt wie der erste römische Kaiser. ${ }^{1}$ Wenige werden sich Dietmar Kienasts Urteils in seinem Standardwerk zu Augustus anschließen wollen, dass Augustus der selbstloseste Herrscher der Geschichte gewesen sei. ${ }^{2}$ Ebenso mutig ist das Urteil von Helmut Halfmann, dass der große Gegner des Augustus, Marcus Antonius, zumindest in charakterlicher Hinsicht der bessere Kaiser geworden wäre, wenn er sich gegen seinen Widersacher durchgesetzt hätte. ${ }^{3}$

Diese beiden Ansichten zeigen schon, dass die Bewertung des ersten Princeps in heutiger Zeit stark davon abhängig ist, wie man zu Antonius, aber auch Caesar, Pompeius oder Cicero steht. Ebenso prägend hat sich die eigene Zeit auf so manche althistorische Charakterisierung ausgewirkt. So ist es nicht dem Zufall zuzuschreiben, dass die erste umfassende augustuskritische Monographie „The Roman Revolution“ von Sir Ronald Syme in erster Auflage 1939 erschienen ist, auch wenn der große Althistoriker in seinem Vorwort angibt, seine Sicht auf die Entstehung des Principats sei von Sallust, Tacitus und insbesondere Asinius Pollio beeinflusst worden. ${ }^{4}$

Natürlich haben die antiken Zeugnisse ebenfalls ihren Anteil am heutigen Augustus-Bild. Nach Tacitus herrschte direkt nach dem Tod des ersten Kaisers Uneinigkeit darüber, wie dessen Leben und Herrschaft einzuschätzen seien. ${ }^{5}$ Während die Kritiker ihm Herrschsucht vorwarfen, hielten ihm andere zugute, dass seine Alleinherrschaft das einzige wirksame Heilmittel für das Vaterland gewesen sei. In der vorangehenden Triumviratszeit habe Augustus, noch unter dem Namen Octavian, dagegen des Häufigeren Antonius und Lepidus nachgeben müssen. Die Haltung des Tacitus zu diesen Vorgängen zeigt sich bereits darin, dass die Fürsprecher des Augustus zuerst zu Wort kommen und ihre Argumente weniger Raum einnehmen als diejenigen der ihnen folgenden Kritiker. Während die Taciteische und Syme'sche Sicht die Nachkriegszeit bestimmte, herrscht heute wieder eine insgesamt eher positive Einschätzung des ersten Princeps vor. ${ }^{6}$

So oder so ist allerdings eine deutliche Zäsur zwischen seiner Herrschaft als Triumvir und der darauffolgenden Zeit erkennbar, wie schon Christoph Martin Wieland festgestellt hat:

\footnotetext{
${ }^{1}$ Vgl. E. Pyy, The Semiotics of Caesar Augustus, Bloomsbury Advances in Semiotics, London u.a. 2018, 178: „Augustus remains a constantly changeable chameleon whose incredible post-mortem fame is based as much on the fluidity of his charakter as on his political and cultural achievement.“

${ }^{2}$ D. Kienast, Augustus. Prinzeps und Monarch, Darmstadt ${ }^{3} 1999,517$ : „Wenn jemand in der Geschichte, so hat Augustus die Macht nicht um ihrer selbst willen und zur Befriedigung seiner persönlichen Bedürfnisse geübt.“

${ }^{3}$ H. Halfmann, Marcus Antonius, Gestalten der Antike, Darmstadt 2011, 229: „Antonius wäre [...] der Ehre, als erster römischer Kaiser in die Geschichte einzugehen, würdig gewesen. Als Mensch und Charakter hätte er den Sieger von Actium und ersten Prinzeps, Augustus, übertroffen, und ihm wäre das zuteil geworden, was jenem bis heute versagt geblieben ist: Begeisterung oder gar Zuneigung.“

${ }^{4}$ R. Syme, The Roman Revolution, 2., verb. Aufl., Oxford 1952, vii.

${ }^{5}$ Tac. ann. 1,9f.

${ }^{6}$ Vgl. z.B. die Beiträge in der Aufsatzsammlung E. Baltrusch/Chr. Wendt (Hg.), Der Erste. Augustus und der Beginn einer neuen Epoche, Darmstadt 2016.
} 
„Wer, der die Begebenheiten der fünfzehn Jahre seines Triumvirats, unter dem Namen Octavianus, und die Geschichte der übrigen zwey und vierzig Jahre seiner Regierung, in einem anderen Buche unter dem Namen Augustus gelesen hätte, könnte sich vorstellen, daß er das Leben einer und ebenderselben Person gelesen habe?“7

Manch einer, und eben auch Wieland, ${ }^{8}$ möchte die ultima verba des Augustus, seine Frage an Freunde, ob er das Schauspiel des Lebens passabel gespielt habe, ${ }^{9}$ so auffassen, dass der Princeps sich anders als der Triumvir hervorragend verstellt habe. Andere sehen hierin einen der stoischen Philosophie verpflichteten Ausspruch. ${ }^{10}$ Selbst wenn man Wieland folgte, ist der Historizität der sogenannten letzten Worte von bekannten Persönlichkeiten naturgemäß zu misstrauen. Gegner des Augustus könnten sie ihm in den Mund gelegt haben.

Folgen wir den Selbstaussagen des Princeps in seinem Tatenbericht ist natürlich keine Veränderung in seiner Machtausübung während der Dreier-, dann Zweier- und letztlich Alleinherrschaft erkennbar: Seine Handlungen waren durchweg selbstlos. Ein derartiges Bild wird auch von den Augustus nahestehenden Dichtern und Geschichtsschreibern vermittelt.

Wie aber wird Augustus in den übrigen Schriften im frühen Principat beurteilt? Wenn es nicht die Kunst der Verstellung war, die aus dem skrupellosen Gewinner der Bürgerkriege den Friedensfürsten machte: Lag es vielleicht daran, dass nach Actium keine kritische Geschichtsschreibung mehr möglich war? Tatsächlich gib es verschiedene direkte und indirekte Hinweise darauf, dass Kritik an der Herrschaft des Augustus und dem Kaiserhaus im Allgemeinen negative Auswirkungen nach sich ziehen konnte.

Hier kommt die im Titel dieses Beitrags an erster Stelle genannte Person in Spiel: C. Asinius Pollio. ${ }^{11}$ Pollio wurde im Jahre 76 v.Chr. geboren und war ein typischer ,Aufsteiger' der Triumviratszeit. Sein Großvater besaß noch nicht einmal das römische Bürgerrecht, während sein Vater immerhin Angehöriger des römischen Ritterstandes war, in Rom lebte und offensichtlich große Sorgfalt bei der Erziehung seines Sohnes aufwandte. Bereits früh erregte Pollio Aufsehen als Prozessredner in Rom gegen Anhänger des Pompeius. ${ }^{12}$ Seine weitere Karriere wurde von Caesar gefördert, auf dessen Seite sich Pollio im Bürgerkrieg schlug. Glücklicherweise besitzen wir verschiedene Selbstäußerungen des Mannes, da sich drei seiner Briefe an Cicero erhalten haben. Zum Anschluss an Caesar schreibt Pollio am 16.3.43 v.Chr.:

\footnotetext{
${ }^{7}$ Chr. M. Wieland, Horazens Briefe aus dem Lateinischen übersetzt, Zweyter Theil, Dessau 1782, 7.

${ }^{8}$ Wieland (Anm. 5) 8: „Nun, sagte er zu seinen umstehenden Vertrauten: Dünkt euch, daß ich den Mimus des Lebens leidlich gespielt habe? Augustus hätte sich nicht deutlicher über das, was wir von seinen so hoch gepriesenen Tugenden zu denken haben, erklären können als durch diesen Ausdruck.“

${ }^{9}$ Suet. Aug. 99,1; vgl. Cass. Dio 56,30,3f.

${ }^{10}$ Kienast (Anm. 2) 148 Anm. 232.

${ }^{11}$ Siehe zum Folgenden P. Groebe, C. Asinius (Nr. 25) Pollio. In: RE II 2 (1896) 1589-1602; C. W. Mendell, The Epic of Asinius Pollio, YClS 1 (1928) 195-207; J. André, La vie et l'oeuvre de C. Asinius Pollio, Études et Commentaires 8, Paris 1949; B. Haller, Asinius Pollio als Politiker und zeitkritischer Historiker. Ein Beitrag zur Geschichte des Überganges von der Republik zum Prinzipat in Rom, 60-30 v.Chr., Diss. Münster 1967; G. Zecchini, Asinio Pollione: Dall'attività politica alla riflessione storiografica. In: ANRW II 30/2, 1982, 1266-1296; A. Drummond, Nr. 56: C. Asinius Pollio. In: T. J. Cornell (Hg.), The Fragments of the Roman Historians I: Introduction, Oxford 2013, 430-445, hier: 430-435.

${ }^{12} \mathrm{Zu}$ Pollio als Redner siehe jetzt M. B. Roller, Losing to Cicero. Asinius Pollio and the Emergence of New Arenas of Competitive Eloquence under Augustus. In: K.-J. Hölkeskamp/H. Beck (Hg.), Verlierer und Aussteiger in der ,Konkurrenz unter Anwesenden‘. Agonalität in der politischen Kultur des antiken Rom, Stuttgart 2019, 189-205.
} 
„Da ich nicht neutral bleiben konnte, habe ich mich, weil ich auf beiden Seiten erbitterte Feinde hatte, der Partei entzogen, bei der ich, wie ich wusste, den Nachstellungen eines meiner Feinde einfach schutzlos preisgegeben sein würde. [...] Caesar aber behandelte mich auf der Höhe seines Glücks, wiewohl er mich eben erst kennengelernt hatte, wie einen ganz alten Vertrauten. Darum habe ich an ihm persönlich mit aller Liebe und Treue gehangen. Wo ich nach eigener Überzeugung verfahren durfte, habe ich so gehandelt, dass jeder anständige Mensch völlig damit einverstanden sein konnte; was mir befohlen wurde, habe ich dann so ausgeführt, dass man sah, wie ungern ich es tat." ${ }^{\text {13 }}$ (Übers. hier und im Folgenden nach H. Kasten)

Derartiges konnte man nach Caesars Ermordung häufiger von dessen vormaligen Gefolgsleuten hören: Man habe die Person zwar geliebt und bewundert, ihre Handlungen aber schon vor den Iden des März verurteilt oder nicht mitgetragen. ${ }^{14}$ Tatsächlich war Asinius Pollio aber anscheinend ein fähiger und loyaler Anhänger Caesars, der diesem gegenüber keinerlei Kritik zum Ausdruck brachte - jedenfalls nicht zu dessen Lebzeiten. So wurde er 45 v.Chr., obwohl erst 31 Jahre alt, mit der Praetur belohnt, welche zu Zeiten der Republik im Regelfall frühestens mit 40 Jahren bekleidet werden konnte. Direkt im Anschluss übernahm er die Statthalterschaft des jenseitigen Spanien, also derjenigen Provinz, welche auch Caesar nach seiner Praetur für sich so gewinnbringend verwaltet hatte. Pollio führte hier den Kampf gegen die Gegner Caesars an, die sich unter dem Pompeius-Sohn Sextus neu formiert hatten. Einen durchschlagenden Erfolg konnte er allerdings nicht erzielen.

Nach der Ermordung Caesars, als Cicero in Rom den Senat in einen Krieg gegen Antonius steuerte, waren die Statthalter und Truppen in den gallischen und spanischen Provinzen von entscheidender Bedeutung. ${ }^{15}$ Die Caesarmörder Brutus und Cassius benötigten Zeit, um den Osten und die dortigen Truppen unter ihre Kontrolle zu bringen. Im Westen standen dagegen ehemals treue Gefolgsleute Caesars, die nach der Ermordung ihres Patrons die Entwicklung in Rom genau verfolgten. Ihre Truppen waren kampferprobt und konnten im Falle einer Einigung entscheidend sein. In Oberitalien hatte sich der Caesarmörder Decimus Brutus in Mutina verschanzt, wo er von Antonius belagert wurde. In Antonius' Rücken sammelten die ehemaligen Caesarianer Hirtius und Pansa zusammen mit Octavian Truppen gegen ihn. In Gallia Narbonensis und Hispania Citerior saß Lepidus, in Gallia Comata Plancus und im jenseitigen Spanien eben Pollio mit drei Legionen unter seinem Kommando, die ausschlaggebend sein konnten. Plancus versicherte, dem Senat ergeben zu sein. Lepidus votierte für Frieden, ließ seine Sympathien für Antonius aber durchblicken. Was tat Pollio? Seine Briefe an Cicero werden von der Forschung immer wieder als Beispiele für den republikanischen und friedliebenden Charakter des Mannes angeführt:

\footnotetext{
${ }^{13}$ Cic. fam. 10,31,2f.: cum vero non liceret mihi nullius partis esse quia utrubique magnos inimicos habebam, ea castra fugi in quibus plane tutum me ab insidiis inimici sciebam non futurum. [...] Caesarem vero, quod me in tanta fortuna modo cognitum vetustissimorum familiarium loco habuit, dilexi summa cum pietate et fide. quae mea sententia gerere mihi licuit ita feci ut optimus quisque maxime probarit; quod iussus sum eo tempore atque ita feci ut appareret invito imperatum esse. (Ed. hier und im Folgenden D. R. Shackleton Bailey, Cambridge 1977).

${ }^{14}$ Siehe z.B. Cic. fam. 11,28,2 (Matius).

${ }^{15}$ Siehe zum Folgenden K. Matijević, Marcus Antonius. Consul - Proconsul - Staatsfeind. Die Politik der Jahre 44 und 43 v.Chr., Osnabrücker Forschungen zu Altertum und Antike-Rezeption 11, Rahden/Westf. 2006, 211-370.
} 
„Charakter und Neigung lassen mich Frieden und Freiheit (libertas) wünschen. Deshalb habe ich auch den Ausbruch des Bürgerkrieges stets tief bedauert. [...] Die ganz unverdiente Missgunst, der mein Tun begegnete, musste mir eine Lehre sein, wie schön die Freiheit und wie elend das Leben unter einer Gewaltherrschaft (dominatio) ist.“16

Pollio betonte, Frieden und Freiheit, womit politische Freiheit gemeint ist, zu favorisieren. Die ihm als Günstling des Dictators entgegengebrachte Missgunst habe ihn davon überzeugt, dass eine politische Karriere in einem freien römischen Staat der seinen vorzuziehen sei. Folglich versprach Pollio unentwegt, sich den Anweisungen des Senats $\mathrm{zu}$ fügen und dass seine Freundschaft zu Antonius der Vergangenheit angehörte. Doch nicht alle trauten in dieser Angelegenheit seinen Worten: Der Caesarmörder Decimus Brutus bemerkte in einem Schreiben an Cicero am 49.4.43 v.Chr.:

„Vor allem bitte ich Dich, den Windhund Lepidus zu bearbeiten; sonst könnte er sich mit Antonius vereinigen und den Krieg gegen uns wieder aufnehmen. Was Asinius Pollio im Schilde führt, ist Dir wohl klar."

Mit den Worten des großen Cicero-Kenners Shackleton Bailey: „Pollio would join Antony if he got the chance. ${ }^{" 18}$ Wobei hiermit natürlich lediglich gemeint ist, dass dies die Meinung des Decimus Brutus war. Der zu Zeiten des Kaisers Tiberius schreibende Velleius Paterculus bescheinigte Pollio in dieser Situation des Jahres 43 eine durch und durch ,iulianische“ Einstellung. ${ }^{19}$ Octavian aber kämpfte noch auf Seiten des Senats gegen Antonius.

Pollio hielt sich tatsächlich abwartend alle Möglichkeiten offen. Solange es so schien, als würde Antonius besiegt, versicherte er Cicero seine Loyalität gegenüber dem Senat. ${ }^{20}$ Nachdem Antonius aber nicht zu bezwingen war und dieser nicht nur Lepidus, sondern auch Plancus für sich gewinnen konnte, dürfte es niemanden überrascht haben, dass sich Pollio kurz darauf im August des Jahres 43 ebenfalls den Truppen des Antonius anschloss. Pollios Beteuerung gegenüber Cicero kurz zuvor, ${ }^{21}$ dass er eine Alleinherrschaft von wem auch immer bekämpfen werde, erscheint unter diesen Umständen hohl und wenig glaubwürdig. Dennoch werden diese Worte immer wieder herangezogen, um eine spätere Unabhängigkeit Pollios im augusteischen Principat zu untermauern. ${ }^{22}$

\footnotetext{
${ }^{16}$ Cic. fam. 10,31,2f. vom 16.3.43 v.Chr.: natura autem mea et studia trahunt me ad pacis et libertatis cupiditatem. itaque illud initium civilis belli saepe deflevi. [...] cuius facti iniustissima invidia erudire me potuit quam iucunda libertas et quam misera sub dominatione vita esset.

${ }^{17}$ Cic. fam. 11,9,1: in primis rogo te ad hominem ventosissimum, Lepidum, mittas, ne bellum nobis redintegrare possit Antonio sibi coniuncto. nam de Pollione Asinio puto te perspicere quid facturus sit.

${ }^{18}$ Epistulae ad familiares 2, hg. und komm. v. D. R. Shackleton Bailey, Cambridge 1977, 523.

${ }^{19}$ Vell. 2,63,3: Pollio firmus proposito et Iulianis partibus fidus, Pompeianis aduersus. (Ed. hier und im Folgenden J. Hellegouarc'h, Paris 1982). - „Asinius Pollio dagegen blieb fest in seiner Haltung, ein Freund der julianischen und ein Gegner der pompejanischen Partei.“ (Übersetzt hier und im Folgenden von M. Giebel).

${ }^{20}$ Siehe Matijević (Anm. 15) 346-370.

${ }^{21}$ Cic. fam. 10,31,3 vom 16.3.43 v.Chr.: Ita, si id agitur ut rursus in potestate omnia unius sint, quicumque is est, ei me profiteor inimicum; nec periculum est ullum quod pro libertate aut refugiam aut deprecer. - „Wenn es also darauf hinausläuft, dass wieder alles in die Hand eines einzigen kommt, mag er sein, wer er will, dann bekenne ich mich als seinen Gegner, und es gibt keine Gefahr, der ich mich für die Freiheit entziehen oder versagen werde."

${ }^{22}$ Syme (Anm. 4) 5f. Siehe unter den vielen Gelehrten, die Syme folgen, nur W. Speyer, Büchervernichtung und Zensur des Geistes bei Heiden, Juden und Christen, Bibliothek des Buchwesens 7, Stuttgart $1981,58,62$.
} 
Nach der Gründung des Triumvirats Ende 43 v.Chr. war Pollio für Antonius tätig, als Statthalter und Heerführer. Er vermittelte zwischen seinem Patron und Octavian in Brundisium im Jahre 40, wofür er im selben Jahr mit dem Consulat belohnt wurde. Vergil besingt dieses in seiner berühmten 4. Ekloge. ${ }^{23} 39$ v.Chr. besiegte Pollio als Proconsul auf dem Balkan (Illyricum oder Macedonien) das Volk der Parthiner, wofür er einen Triumph am 25.10.39 oder 38 v.Chr. feiern durfte. Danach hat sich Pollio aus der Politik zurückgezogen und sich insbesondere der Geschichtsschreibung gewidmet. Der homo novus hatte alles erreicht, was für ihn politisch zu erreichen war. Einen riskanten Platz in der ersten Reihe strebte er nicht an.

Die Beute aus seinem Feldzug nutzte er, um die erste öffentliche Bibliothek in Rom einzurichten, und zwar in dem von ihm restaurierten Atrium Libertatis. Hierin möchte beispielsweise Llewelyn Morgan eine Demonstration Pollios erkennen können: Politische Freiheit sei nun auf die Literatur beschränkt gewesen. ${ }^{24}$

Als sich eine alles entscheidende Auseinandersetzung zwischen Antonius und Octavian in den 30er Jahren v.Chr. anbahnte, versuchten beide Kontrahenten, möglichst viele einflussreiche Senatoren auf ihrer Seite zu versammeln. Wohl in dieser Zeit hat Pollio eine Schrift mit dem Titel contra maledicta Antonii verfasst, deren Inhalt unbekannt ist. Angenommen wird, dass Antonius die Gefolgschaft des Pollio einforderte und an frühere Wohltaten erinnerte, wogegen Pollio sich zur Wehr setzte. Vor der Schlacht bei Actium ist Pollio dann von Octavian aufgefordert worden, sich dessen Heer anzuschließen, was Pollio mit dem Hinweis auf die früheren Freundschaftsdienste von und für Antonius ablehnte. ${ }^{25}$ Dass er sich zur Beute des Siegers erklärte, spiegelt seine grundsätzliche politische Haltung m.E. treffender wieder als seine frühere Aussage gegenüber Cicero, er werde eine dominatio unter allen Umständen bekämpfen. ${ }^{26}$ Der Ausgang der Schlacht ist bekannt.

Wie aber gestaltete sich das Verhältnis zum Alleinherrscher Augustus? Zwei Forschungsansichten stehen sich in dieser Frage gegenüber: Einerseits wird im Anschluss an Ronald Syme Asinius Pollio als traditioneller Verfechter der Republik angesehen, der in Opposition zu Augustus stand und aufgrund seines Sozialprestiges nicht mundtot gemacht werden konnte. ${ }^{27}$ Andererseits hat Albert Bosworth in einem vielbeachteten Aufsatz darzulegen versucht, dass Pollio sich relativ offen äußern durfte, weil er ein Freund des Augustus geworden war. ${ }^{28}$

Zumindest in der Triumviratszeit kam es noch zum offenen Schlagabtausch zwischen den beiden. Octavian soll Spottverse über Pollio verfasst haben, worauf dieser sagte:

„Doch ich schweige, denn es ist nicht leicht, gegen den zu schreiben, der einen auf die Todesliste schreiben kann“ ${ }^{29}$ (Übers. von K. Bringmann/D. Wiegandt)

\footnotetext{
${ }^{23}$ Verg. ecl. 4,1-17.

${ }^{24}$ L. Morgan, The Autopsy of C. Asinius Pollio, JRS 90 (2000) 51-69, hier: 66.

${ }^{25}$ Vell. 2,86,3: rogante Caesare ut secum ad bellum proficisceretur Actiacum: ,Mea, inquit, in Antonium maiora merita sunt, illius in me beneficia notiora; itaque discrimini uestro me subtraham et ero praeda uictoris. “ „Caesar (Octavian) bat Pollio nun, mit ihm in den Kampf um Actium zu ziehen. Pollio aber sagte: ,Meine Verdienste gegenüber Antonius sind zu groß, seine Gunstbeweise gegen mich zu bekannt; daher werde ich mich eurem Streit entziehen und die Beute des Siegers werden."“

26 Ähnlich Drummond (Anm. 11) 432.

27 So z.B. auch Speyer (Anm. 22) 58, 62.

${ }^{28}$ A. B. Bosworth, Asinius Pollio and Augustus, Historia 21, 1972, 441-473; ihm folgt Drummond (Anm. 11) $434 f$.

${ }^{29}$ Macr. Sat. 2,4,21: At ego taceo. Non est enim facile in eum scribere, qui potest proscribere (Ed. K. Bringmann/D. Wiegandt, Darmstadt 2008).
} 
Geschwiegen hat Pollio somit eigentlich nicht. Während des augusteischen Principats hat er sogar bei einer Gelegenheit im Senat das Wort gegen die von Augustus geförderten Troiaspiele ergriffen, weil sein Enkel sich hierbei verletzt hatte. ${ }^{30} \mathrm{Zu}$ diesem Zeitpunkt muss Pollio schon hochbetagt gewesen sein.

Dass er sich noch als 80jähriger einiges herausnehmen konnte, belegt eine bei Seneca d.Ä. überlieferte Episode, ${ }^{31}$ wonach Augustus sich beim Tod seines Enkels Gaius Caesar darüber beklagte, dass Pollio ein Festmahl abhielt, woraufhin dieser lapidar bemerkte, dass er auch beim Tod seines eigenen Sohnes Essen zu sich genommen habe.

In privaten Dingen hat sich Pollio im Principat also eine gewisse Eigenständigkeit bewahrt. Witzige, sogar bissige Bonmots musste nicht nur Augustus ertragen. Literaturkritische oder sonstige negative Kommentare erduldeten Sallust, Livius, Caesar, Cicero und Plancus; die meisten der Genannten aber erst nach ihrem Tod. In politischer Hinsicht hat Pollio nach Actium dagegen keine von Augustus unabhängige Position einzunehmen gesucht. Jedenfalls wissen wir hiervon nichts. Kam es hart auf hart, war Pollio ferner bereit, sich dem Befehl des Princeps unterzuordnen. So geschehen im Falle des Timagenes von Alexandria, einem Geschichtsschreiber, der mit Augustus in Konflikt geriet und von Pollio aufgenommen wurde. ${ }^{32}$ Als der Kaiser ihn darauf ansprach, bot Pollio an, Timagenes des Hauses zu verweisen, wenn es ihm befohlen werde, wozu es aber nicht kam. Interessanterweise erhielt Timagenes erst Zugang zum Hause Pollios, nachdem er mit seinem vormaligen Patron Augustus gebrochen hatte. Davor waren die beiden Historiographen verfeindet, was ein vielsagendes Licht auf Pollio wirft.

Bezeichnend ist, dass Asinius Pollio noch nach seinem Tode von Velleius Paterculus, einem euphorischen Anhänger des Principats, in den höchsten Tönen gelobt wurde, und zwar mehrfach. Auch dies spricht gegen eine politische Opposition. Insbesondere im Vergleich mit Munatius Plancus, der wegen wechselnder Loyalität in der Triumviratszeit von Velleius harsch kritisiert wurde, fällt auf, dass Pollio sehr gut wegkommt, obgleich er sich, wie an seinem erwähnten Brief demonstriert, bei wichtigen politischen Entscheidungen ebenfalls gerne zurückhielt, bis er sich sicher sein konnte, auf der Seite des Siegers zu stehen. Nichtsdestotrotz ist Pollio gewiss auch kein Anhänger des Augustus gewesen. Während Plancus 27 v.Chr. vorgeschlagen hat, Octavian den Ehrentitel Augustus zu verleihen, und 22 v.Chr. die Zensur ausüben durfte, erhielt Pollio keinerlei politische Aufgabe oder Ehrung unter Augustus. Allerdings wurde sein einziger überlebender Sohn, Asinius Gallus, im frühestmöglichen Alter von 33 Jahren 8 v.Chr. Consul. Zudem durfte Gallus die Tochter Agrippas, Vipsania, ehelichen. Folglich besitzt das Urteil Senecas, dass die Familie des Asinius zu den Gewinnern der augusteischen Herrschaft zählte, einige Berechtigung. ${ }^{33}$

\footnotetext{
${ }^{30}$ Suet. Aug. 43,2: sed et Troiae lusum edidit frequentissime maiorum minorumque puerorum, prisci decorique moris existimans clarae stirpis indolem sic notescere. [...] mox finem fecit talia edendi Asinio Pollione oratore grauiter inuidioseque in curia questo $A<e>$ sernini nepotis sui casum, qui et ipse crus fregerat. (Ed. M. Ihm, Leipzig 1908) - „Ja sogar das Trojaspiel ließ er [Augustus] sehr häufig von kleineren und größeren Jungen aufführen, da er darin eine alte und ehrenvolle Sitte sah und glaubte, dass die Nachkommen einer berühmten Familie so bekannt würden. [...] Bald aber hörte er auf mit Veranstaltungen dieser Art, weil sich der Redner Asinius Pollio im Senat heftig und voller Entrüstung darüber beklagte, dass sein Enkel Aeserninus gestürzt sei, der hatte sich dabei sogar noch ein Bein gebrochen.“ (Übers. nach H. Martinet).

${ }^{31}$ Sen. contr. 4 praef. 5 .

${ }^{32}$ Sen. de ira $3,23,7 f$.

${ }^{33}$ Sen. de Clem. 1,10,1.
} 
Wie nun hat sich Pollio in seinem historiographischen Werk, den Historien, über Octavian geäußert? Da sich die Schrift nicht erhalten hat, sind wir auf Vermutungen angewiesen. Bevor wir uns dieser Frage widmen, müssen wir zuerst über Abfassungszeit und Inhalt der Historien sprechen. Einige Informationen ergeben sich aus einem Pollio gewidmeten Gedicht des Horaz:

„Den Bürgerzwist, der sich entspann, als Metellus Consul war des Krieges Quellen, Fehler, Wechsel,

Fortunens Spiel, den unglücksvollen

Feldherrnbund und Waffen, noch immer von ungesühntem Blut befleckt, beschreibst du jetzt, mein Pollio, ein Werk, reich an Gefahr! Und wandelst über Feuer dahin.

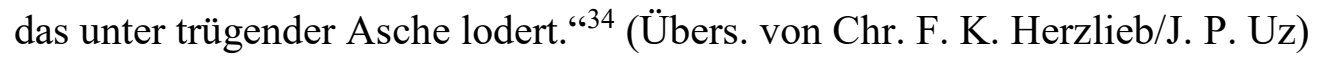

Pollios Historien setzten also im Jahre 60 v.Chr. ein, zur Zeit des Consuls Metellus Celer, als es zwischen Caesar, Pompeius und Crassus zur Gründung des sog. 1. Triumvirats kam, welches augenscheinlich als Beginn der Bürgerkriegszeit angesehen wird. Sehr viel mehr scheint der Dichter nicht zu wissen, da Pollio, wie es im weiteren Verlauf des Gedichtes heißt, noch mit der Abfassung seines Werkes beschäftigt war. Horaz warnt nun aber seinen Freund ganz ausdrücklich: Mit seinem Werk und der ihn ihm geschilderten Zeit wandle Pollio auf sehr gefährlichem Grund. Richard Heinze schreibt in seinem Kommentar zu den von Horaz gewählten Worten:

„Dies Bild ist völlig schief, wenn es nicht meint, daß der Historiker selbst Gefahr läuft, sich zu ,verbrennen'. Die damals entfachten Leidenschaften glimmen noch fort[,] und es ist daher ein Risiko, insbesondere für einen als freimütig bekannten Mann wie Pollio, jene Zeiten zu schildern. “35

Ob die Warnung irgendeinen Einfluss auf den Endpunkt der Historien Pollios hatte, muss offenbleiben. Relativ sicher ist, dass die Ereignisse bei Philippi 42 v.Chr. noch behandelt wurden, da ein Pollio-Fragment Brutus und Cassius erwähnt (siehe unten). Wir wissen ferner, dass die Darstellung insgesamt 17 Bücher umfasste. ${ }^{36}$ Dass jedes Buch ein Jahr beschrieb, also den Zeitraum von 60 bis 42 v.Chr., und Pollio bewusst die Zeit danach bis Actium aussparte, um sich zu schützen, ist eine von der Forschung diskutierte Möglichkeit, ${ }^{37}$ die darauf basiert, dass Pollio sich von der Warnung des Dichters beeindrucken ließ. Wesentlich wahrscheinlicher ist allerdings, dass Pollio seinen eigenen Consulat und die daran anschließende Statthalterschaft in den Jahren 40 und 39 v.Chr. ebenfalls behandelt hat. Dass die komplette Bürgerkriegszeit bis zur Schlacht bei Actium 31 v.Chr. und sogar noch Ereignisse danach berücksichtigt wurden, ist keinesfalls auszuschließen. ${ }^{38}$ Sueton teilt mit, dass der Grammatiker Ateius Philologus zuerst Sallust behilflich war und nach dessen Tod Asinius Pollio verschiedene

\footnotetext{
${ }^{34}$ Hor. c. 2,1,1-8: Motum ex Metello consule civicum / bellique causas et vitia et modos / ludumque Fortunae gravisque / principum amicitias et arma / nondum expiatis uncta cruoribus, / periculosae plenum opus aleae, / tractas et incedis per ignis / suppositos cineri doloso. (Ed. F. Klingner, Leipzig 1959). ${ }^{35}$ Q. Horatius Flaccus, 1. Teil: Oden und Epoden, erklärt v. A. Kiessling, 8. Aufl. v. R. Heinze, mit einem Nachwort und bibliographischen Nachträgen v. E. Burck, Berlin 1955, 164.

${ }^{36}$ Suda s.v. Asinnios Polion.

37 Syme (Anm. 4) 6.

${ }^{38}$ Vgl. Drummond (Anm. 11) 438f.
} 
Hinweise für das Abfassen von historischen Texten gegeben habe. ${ }^{39}$ Somit hat Pollio wahrscheinlich bereits 35 v.Chr., dem Todesjahr Sallusts, an seinen Historien gearbeitet. Leider lässt sich das erwähnte Gedicht von Horaz nicht näher datieren. Es wurde zwar 23 v.Chr. publiziert, die Abfassungszeit ist aber umstritten und könnte nach Meinung mancher Gelehrter noch in die dreißiger Jahre fallen. Man kann somit festhalten, dass Pollio spätestens um die Mitte der 30er Jahre angefangen hat, an seinen Historien zu arbeiten, und dass er die Schrift unter Umständen bis weit in die 20er Jahre hinein fortführte. Der behandelte Zeitraum reichte von $60 \mathrm{v}$.Chr. bis wenigstens 39, vielleicht sogar bis $31 \mathrm{v}$.Chr. und darüber hinaus. ${ }^{40}$ Wir können also nicht ausschließen, dass die Alleinherrschaft des Augustus ohne jeden Einfluss auf Pollios Geschichtswerk blieb.

Die generelle Tendenz seiner Schrift ist nach Ansicht von Ronald Syme und vielen ihm folgenden Forschern ,republikanisch“ gewesen. Wie bereits erwähnt, werden in dieser Hinsicht immer wieder die insgesamt drei Schreiben Pollios an Cicero angeführt. Darüber hinaus behauptete der Geschichtsschreiber Cremutius Cordus, dass Pollio die Caesarmörder Brutus und Cassius positiv dargestellt habe. ${ }^{41}$ Letzteres bedeutet aber natürlich nicht, dass Pollio die Seite der Caesarmörder favorisiert haben muss, er selbst stand ja auf der anderen. Ebenso gut könnte der Historiograph versucht haben, einen möglichst objektiven Standpunkt einzunehmen. Für eine ausgewogene Sicht spricht nicht nur Pollios eigene Rolle in den Ereignissen, sondern auch seine harsche Cicero-Kritik. $\mathrm{Zu}$ dem großen Redner bemerkt er in der einzigen längeren, wörtlich erhaltenen Textpassage seiner Historien:

„Hätte er [Cicero] es nur vermocht, das Glück maßvoller und das Unglück mutiger zu ertragen! In beiden Fällen nämlich glaubte er, eine Änderung sei nicht mehr möglich. So ballten sich schwere Hass-Gewitter gegen ihn zusammen, wobei ihn seine Feinde umso mutiger angriffen, weil er Feindschaften mit mehr Entschlossenheit aufnahm als durchhielt [...]. Ich selbst würde nicht einmal sagen, sein Lebensende sei jämmerlich gewesen, hätte er nicht selbst seinen Tod als so elend empfunden. “42 (Übers. nach O. und E. Schönberger)

Darüber hinaus unterstellte Pollio seinem ehemaligen Patron Caesar, dass dessen Bericht über die Gallischen Kriege zum Teil auf falschen Aussagen basierte. ${ }^{43}$ Die Tendenz der Historien wird also weder ausschließlich ,republikanisch' noch ganz und gar ,caesarianisch ' gewesen sein. ${ }^{44}$ Keine Hinweise geben die überlieferten Fragmente auf die Position der Historien in der Auseinandersetzung zwischen Antonius und Octavian.

Diejenigen Forscherinnen und Forscher, die auf Grundlage der bisher diskutierten Details aus dem Leben und dem Werk Pollios auf eine generelle Tendenz seines

\footnotetext{
${ }^{39}$ Suet. gramm. 10,6.

${ }^{40}$ Vgl. auch Morgan (Anm. 24) 54 mit Anm. 18.

41 Tac. ann. 4,34,2-4.

${ }^{42}$ Sen. suas. 6,24: Utinam moderatius secundas res et fortius adversas ferre potuisset! Namque utraeque cum evenerant ei, mutari eas non posse rebatur. Inde sunt invidiae tempestates coortae graves in eum certiorque inimicis adgrediendi fiducia; maiore enim simultates adpetebat animo quam gerebat. Sed quando mortalium nulli virtus perfecta contigit, qua maior pars vitae atque ingenii stetit, ea iudicandum de homine est. [...] Atque ego ne miserandi quidem exitus eum fuisse iudicarem, nisi ipse tam miseram mortem putasset. (Ed. M. Winterbottom, Cambridge 1974).

${ }^{43}$ Suet. Iul. 56,4.

${ }^{44}$ Zur generellen Problematik der Begriffe ,republikanisch`oder auch ,caesarianisch` siehe K. Matijević, The Caesarian Opposition against Mark Antony after the Ides of March. In: R. Cristofoli/A. Galimberti/F. Rohr (Hg.), Lo spazio del non-allineamento a Roma fra Tarda Repubblica e Primo Principato. Forme e figure dell'opposizione politica, Atti del Convegno die Studi Milano 11-12 Aprile 2013, Centro ricerche e documentazione sull'antichità classica, Monografie 36, Mailand 2014, 41-58.
} 
Werkes geschlossen haben, sind in einem zweiten Schritt dazu übergegangen, im Rahmen der klassischen Quellenforschung, den Anteil der Historien an den überlieferten Schilderungen der Triumviratszeit festzumachen, insbesondere in den Schriften Appians, Cassius Dios, Plutarchs und Suetons. So hat Emilio Gabba in den Fußstapfen Ronald Symes eine prorepublikanische Tendenz identifiziert und Pollio zur Hauptquelle der Bürgerkriege Appians deklariert, obgleich letztere bereits mit den Ereignissen des Jahres 133 v.Chr. einsetzen. ${ }^{45}$ Andere, wie Gerhard Dobesch, ${ }^{46}$ meinen, dass die Historien Hauptvorlage der Römischen Geschichte des Cassius Dio gewesen seien, da deren generelle Tendenz überparteilich, oft caesarfreundlich, aber nicht caesarianisch sei und der Senat als in republikanischer Hinsicht untauglich charakterisiert werde. Llewelyn Morgan wiederum erklärt die Historien Pollios sogar zur Hauptquelle von Appian, Dio, Plutarch und Sueton. ${ }^{47}$ Tatsächlich ähneln sich immer wieder einzelne Details und Episoden bei diesen Autoren, nur müssen diese nicht zwingend auf Pollio zurückgehen. Der bereits erwähnte Cremutius Cordus kommt beispielsweise vielfach ebenfalls infrage, zumal Appian in seiner gesamten Darstellung die Historien Pollios nur ein einziges Mal erwähnt, und zwar bezeichnender Weise im Rahmen einer Korrektur der Schriften Caesars. ${ }^{48}$ Natürlich handelt es sich hierbei nicht um einen Gegenbeweis. Eine Stelle bei Plutarch deutet z.B. darauf hin, dass Appian zumindest in einem weiteren Fall die Historien benutzt haben könnte, ohne auf sie explizit hinzuweisen. ${ }^{49}$ In der Tat war es in den antiken Historiographien keineswegs üblich, seine Quellen zu nennen. Christopher Pelling hat allerdings in seinem Kommentar der Caesar-Vita Plutarchs zu bedenken gegeben, dass Appian hier auch Plutarch benutzt haben könnte. ${ }^{50}$

Teile der Forschung gehen in einem dritten Schritt noch weiter und meinen sogar, Ähnlichkeiten in den Darstellungen Plutarchs, Dios und Appians für die Zeit nach Philippi am besten damit erklären zu können, dass Pollio weiterhin Hauptquelle aller drei war. An dieser Stelle ist noch einmal daran zu erinnern, dass wir nicht wissen, wie weit der Inhalt der Historien zeitlich reichte. Sicher ist, wie gesagt, nur, dass die Schlacht von Philippi und sehr wahrscheinlich auch Pollios Consulat und Proconsulat behandelt wurden. Ferner werden von Plutarch und Appian gerade für diese Zeit die Memoiren des M. Valerius Messalla Corvinus als Quelle ausdrücklich erwähnt. ${ }^{51}$

Im Folgenden sei ein typisches Beispiel für die Identifizierung des ursprünglichen Inhalts der pollionischen Historien durch die Forschung angeführt: In Vergils Aeneis $(2,554-558)$ meint man, zuletzt Llewelyn Morgan, ${ }^{52}$ eine Anspielung auf den Tod des Pompeius erkennen zu können. Da auch Servius in seinem Aeneis-Kommentar eine entsprechende Bemerkung macht, ${ }^{53}$ ist diese Ansicht an und für sich erwägenswert. Wenn sich aber alle erhaltenen antiken Ausführungen zum Tod des Pompeius ähneln, ${ }^{54}$

\footnotetext{
${ }^{45}$ E. Gabba, Appiano e la storia delle guerre civili, Biblioteca di cultura 59, Florenz 1956, 83 bzw. Appiani bellorum civilium liber primus, hg., übers. und komm. v. E. Gabba, Biblioteca di studi superiori 37, 2. Aufl., Florenz 1967, xxviii-xxxi.

${ }^{46}$ G. Dobesch, Zu Caesars Sitzenbleiben vor dem Senat und zu der Quelle des Cassius Dio. In: G. Dobesch, Ausgewählte Schriften 1. Römer und Griechen, hg. v. H. Heftner/K. Tomaschitz, Köln/Weimar/Wien 2001, 275-361, hier 322 (Ursprünglich publiziert in: Tyche 3, 1998, 39-102).

${ }^{47}$ Morgan (Anm. 24) 51.

${ }^{48}$ App. civ. 2,82.

${ }^{49}$ Vgl. App. civ. 2,35 mit Plut. Caes. 32.

${ }^{50}$ Plutarch, Caesar, übers. und komm von Chr. Pelling, Oxford 2011, 314.

${ }^{51}$ Plut. Brut. 45; App. civ. 4,112.

${ }^{52}$ Morgan (Anm. 24) 52-55. Er folgt J. L. Moles, Virgil, Pompey and the Histories of Asinius Pollio, CW 76, 1982/1983, 287f.

53 Serv. Aen. 2,557.

${ }^{54}$ Vell. 2,53,1-3; Lucan. 8,698-711; Plut. Pomp. 78-80; App. civ. 2,85f.; Cass. Dio 42,3-5.
} 
muss dies nicht heißen, dass sie sich sämtlich auf die Historien des Asinius Pollio beziehen, nur weil dieser angeblich die Hauptquelle für die Bürgerkriege sei. ${ }^{55}$ Viel einleuchtender ist doch, dass die Details seines Todes sich tatsächlich so abgespielt haben und dass Vergil, der 48 v.Chr. immerhin schon 22 Jahre alt war, selbst hiervon gehört hatte. Hierbei ist auch zu bedenken, dass die verschiedenen Beschreibungen von Ciceros Tod sich ebenfalls sehr ähneln, sich in ihrer Wertung aber von Pollios oben zitierten Worten vollkommen unterscheiden. ${ }^{56}$

Was also sagt uns C. Asinius Pollio über den Umgang mit kritischer Geschichtsschreibung im augusteischen Principat? Tatsächlich nicht viel. Er ist zwar derjenige, der immer wieder dafür angeführt wird, dass eine unabhängige Geschichtsschreibung unter dem ersten Kaiser existiert habe, über seine Historien lässt sich aber wenig sagen. Man vermutet zwar allseits, dass dieses Werk den Inhalt sämtlicher späteren Darstellungen bestimmt habe. Ob dem aber tatsächlich so war, lässt sich nicht mehr sicher feststellen. In den „Fragments of the Roman Historians“" werden für Pollio neben sechs Testimonien nur dreizehn Fragmente aufgeführt. ${ }^{57}$ Falls seine Schilderung der Bürgerkriege ihren Endpunkt mit seinem Triumphzug in 39 v.Chr. gefunden haben sollte, war Pollio in seiner Geschichte nicht gezwungen gewesen, sich zwischen Antonius und Octavian zu entscheiden, da sich der endgültige Bruch zwischen den beiden Triumvirn erst später vollzog.

Außerhalb von seiner Geschichtsschreibung war Pollio politisch nicht mehr aktiv gewesen. Die überlieferten biographischen Details zeigen, dass er sich eines sehr freimütigen Tons gegenüber dem Princeps bediente, ohne Konsequenzen fürchten zu müssen. Einen offenen Bruch hat er allerdings zu keiner Zeit riskiert, weshalb seine Familie keine Nachteile zu befürchten hatte. Im Gegenteil, sein Sohn vollzog unter Augustus eine steile politische Karriere. Geholfen haben mag, dass Pollio zwar nicht dem Kaiser selbst, aber immerhin einigen seiner Freunde, wie Vergil und Horaz, nahestand.

$\mathrm{Zu}$ fragen ist, inwiefern man Pollios Situation verallgemeinern kann. Dieter Timpe hält ihn für einen Einzelfall und stellt Pollios „,notorische Exzentrizität“ heraus, was zumindest im Falle der vorlauten Art Pollios kaum abzustreiten ist. ${ }^{58}$ Andererseits gibt es durchaus vergleichbare Karrieren, wie diejenige des bereits erwähnten M. Valerius Messalla Corvinus: ${ }^{59}$ Wie Pollio wechselte er die Seiten von Antonius zu Octavian. Corvinus war in augusteischer Zeit ebenfalls Consular und Triumphator. Auch er schrieb eine Geschichte über die Bürgerkriege, in der er die Caesarmörder positiv dargestellt haben soll. ${ }^{60}$ Ferner war er wie Pollio Förderer anderer Literaten und Künstler. Allerdings hatte Corvinus im Principat noch politische Ambitionen: 26 oder 25 v.Chr. war er praefectus urbi, um kurz darauf wieder, freiwillig oder unfreiwillig, zurückzutreten. ${ }^{61}$ Dennoch war er es, der im Jahre 2 v.Chr. im Senat den pater patriaeTitel für Augustus beantragte. ${ }^{62}$ Anders als Pollio wird Messalla von Ronald Syme aus diesem Grunde auch politischer Opportunismus unterstellt. ${ }^{63}$

\footnotetext{
55 So aber Morgan (Anm. 24) 52.

${ }^{56}$ Siehe Morgan (Anm. 24) 54 Anm. 18.

${ }^{57}$ A. Drummond, Nr. 56: C. Asinius Pollio. In: T. J. Cornell (Hg.), The Fragments of the Roman Historians II: Texts and Translations, Oxford 2013, 854-867.

${ }^{58}$ D. Timpe, Geschichtsschreibung und Prinzipatsopposition. In: A. Giovannini/D. van Berchem (Hg.), Opposition et résistances à l'empire d'Auguste à Trajan, Entretiens Fondation Hardt 33, Genf 1987, 65102, hier: 71.

${ }^{59}$ Vgl. hierzu Morgan (Anm. 24) 66 Anm. 84.

60 Tac. ann. 4,34,3f.

${ }^{61}$ Tac. ann. 6,11.

${ }^{62}$ Suet. Aug. 58,2.

${ }^{63}$ Syme (Anm. 4) 482.
} 
Neben Pollio und Messalla sind Details zu weniger bekannten Historiographen in augusteischer Zeit überliefert, die freilich ein etwas anderes Licht auf die literarische Freiheit unter dem ersten römischen Kaiser werfen.

Seneca d. Ä. berichtet, ${ }^{64}$ dass Titus Labienus, der insbesondere als Redner eine spitze Zunge besessen haben soll, auch ein Geschichtswerk verfasst hat, aus dem der Autor bestimmte Passagen seinem Publikum beim Vorlesen verschwieg mit dem Hinweis, dass man hierfür auf seinen Tod warten müsse. ${ }^{65}$ Laut Seneca habe Labienus ein Pompeiani spiritus erfüllt, eine pompeianische, wohl also republikanische Gesinnung. Dass er sich ferner üblicherweise nicht gescheut haben soll, gegen alles und jeden zu wettern, wirft auf die Zurückhaltung in Bezug auf sein Geschichtswerk ein besonderes Licht. Letztlich wurden seine Schriften verbrannt, nach Seneca auf Senatsbeschluss. Angeblich sollen die Werke des Mannes, der die Verbrennung verursacht haben soll, noch zu dessen Lebzeiten ebenfalls dem Feuer übereignet worden sein. Dies spricht ebenso wenig gegen Augustus und seinen Kreis als Drahtzieher wie das ebenfalls bei Seneca d.Ä. überlieferte Urteil, dass unter Augustus freie Meinungsäußerung möglich gewesen sei. ${ }^{66}$ Es ist schwer vorstellbar, dass der Princeps den Senatsbeschluss und die Verbrennung der Werke des Labienus nicht abgesegnet haben soll, zumal Cassius Dio berichtet, dass dies unter Augustus kein Einzelfall war. ${ }^{67}$ Dass ein Strohmann die formelle Anklage übernahm, erstaunt wenig.

Ein weiteres Opfer der augusteischen Zensur war Cassius Severus. Von diesem heißt es direkt im Anschluss an die Episode über Labienus bei Seneca, dass er mit diesem verfeindet war. ${ }^{68}$ Dennoch kannte er dessen Werke und machte sich über die Verbrennung derselben lustig, weshalb er wohl nicht der Ankläger des Labienus war, auch wenn dies von einem Teil der Forschung vermutet wird. ${ }^{69}$ Nach Sueton wurden Cassius' Schriften unter Augustus ebenfalls aus dem Verkehr gezogen, ${ }^{70}$ laut Tacitus durch eine lex maiestatis. ${ }^{71}$ Auch hierbei fungierte nicht der Princeps selbst als Ankläger, sondern dessen Anhänger Paullus Fabius Maximus. Cassius Severus wurde nach Kreta verbannt, was ihn aber nicht zum Schweigen brachte. ${ }^{72}$ Er wurde im Jahre 24 n.Chr., also schon unter Kaiser Tiberius, enteignet und musste nach Seriphos ausweichen.

Natürlich kann man nicht ausschließen, dass die genannten Männer gar nicht wegen ihrer Kritik am Principat verfolgt wurden. Vielleicht war es eher die Art und Weise, wie diese Kritik vorgetragen wurde. So bemerkt Tacitus in seinen Historien, dass die Geschichtsschreibung nach Actium nicht nur von Schmeichelei bestimmt wurde, sondern ebenso von Hass auf die Herrschenden und Unwissenheit um den Staat. ${ }^{73}$

\footnotetext{
${ }^{64}$ Sen. contr. 10 praef. $4-8$.

${ }^{65}$ Nicht nachvollziehbar ist die Interpretation von D. Rohmann, Book Burning as Conflict Management in the Roman Empire (213 BCE-200 CE), AncSoc 43, 2013, 115-149, hier: 130: „In fact, Labienus anticipated that the most problematic passages of his history book would become most popular only after his death."

66 Sen. contr. $2,4,13$.

${ }^{67}$ Cass. Dio 56,27,1.

${ }^{68}$ Sen. contr. 10 praef. 8.

${ }^{69}$ V. Rudich, Navigating the Uncertain: Literature and Cencorship in the Early Roman Empire, Arion 14, 2006, 7-28, hier: 24; Drummond (Anm. 11) 472 Anm. 3; Rohmann (Anm. 65) 131.

${ }^{70}$ Suet. Cal. 16.

71 Tac. ann. 1,72.

72 Tac. ann. 4,21 .

73 Tac. hist. 1,1 .
} 
Gerne würde man ferner prüfen, ob es in den immerhin über 40 Jahren Regierungszeit zwischen Actium und Tod des Augustus nicht verschiedene Phasen des Umgangs mit Kritik durch Geschichtsschreiber gab. Dieser Ansatz scheitert allerdings allein schon daran, dass es an sicheren datierenden Details fehlt. Titus Labienus und Cassius Severus scheinen gegen Ende der Regierungszeit des Augustus verfolgt worden zu sein. ${ }^{74}$ Etwa zeitgleich konnte Cremutius Cordus nach Sueton aus seinem Geschichtswerk, welches Brutus und Cassius pries, aber Augustus selbst vorlesen und wurde nicht belangt, ${ }^{75}$ obgleich ein ebenfalls von Sueton überliefertes Fragment seines Werkes den Princeps nicht im besten Licht erscheinen lässt. ${ }^{76}$ Wenige Jahre darauf bildeten die Annalen des Cremutius Cordus (vielleicht in einer nach dem Tod des Augustus überarbeiteten Version? ${ }^{77}$ ) dann wiederum die Grundlage für eine Anklage durch die Häscher des Seian. ${ }^{78}$ Unter Umständen war also unter Augustus der Ton, der ein Geschichtswerk bestimmte, entscheidender als der behandelte Inhalt. Hierauf deutet ebenfalls die Verteidigungsrede des Cremutius Cordus hin, in der dieser darauf aufmerksam macht, dass Livius den Gegner Caesars, Pompeius Magnus, derart positiv dargestellt habe, dass Augustus ihn scherzhaft einen Pompeianer nannte. Cassius Dio sagt ausdrücklich, dass der Ton entscheidender war als der Inhalt. ${ }^{79} \mathrm{Zu}$ berücksichtigen ist aber ebenfalls, dass Augustus selbst Vergil bewegt haben soll, Passagen über Cornelius Gallus aus seinen Gedichten zu entfernen. ${ }^{80}$ Hier spielte offensichtlich allein der Inhalt eine Rolle. Augenscheinlich war der zweite Kaiser, Tiberius, noch dünnhäutiger als sein Stiefvater Augustus. Wenn wir Sueton glauben dürfen, ermahnte Augustus seinen Stiefsohn, kritische Stimmen nicht allzu ernst zu nehmen, wenn sie nicht zu einer Gefahr für ihre Herrschaft werden konnten. ${ }^{81}$

Wer gar kein Risiko eingehen wollte, schwieg allerdings besser gleich, auch unter Augustus. Dass bestimmte Zeugnisse gar nicht erst publiziert worden sind, zeigt der Briefwechsel Ciceros. Sein Freund und Verleger Atticus hat sicherlich nicht ohne Grund darauf verzichtet, seine Antworten an Cicero für sich zu behalten. Über die genaue Ursache kann man nur spekulieren. Viel zu selten wird in dieser Hinsicht aber beachtet, dass beispielsweise von dem Briefwechsel des Jahre 43 v.Chr. zwischen Cicero und Octavian die Erwiderungen des späteren Princeps ebenfalls verloren sind. Syme macht klar, dass dies kein Zufall sein dürfte. ${ }^{82}$ Atticus, dessen Tochter mit Agrippa, einem engen Gefolgsmann des Augustus, verheiratet war, wird bewusst auf

\footnotetext{
${ }^{74}$ Siehe R. A. Bauman, Impietas in Principem. A study of treason against the Roman emperor with special reference to the first century A.D., Münchener Beiträge zur Papyrusforschung und antiken Rechtsgeschichte 67, München 1974, 28-31.

${ }^{75}$ Suet. Tib. 61,3 .

${ }^{76}$ Suet. Aug. 35,2. Siehe hierzu insbesondere H. Tränkle, Zu Cremutius Cordus fr. 4 Peter, MH 37, 1980, 231-241.

${ }^{77}$ So vermutungsweise Bauman (Anm. 74) 103. Dann wären die kritischen Stellen unter Augustus unter Umständen noch gar nicht Teil der Annalen gewesen, was wiederum erklären könnte, warum Augustus nichts zu bemängeln hatte. Doch bleibt dies gänzlich unsicher, wie auch Bauman anmerkt.

${ }^{78}$ Tac. ann. 4,34f. Dass es beim Prozess gegen Cremutius Cordus nicht nur bzw. nicht hauptsächlich um dessen Schriften gegangen sei, denken M. Gelzer, Ti. Iulius (Nr. 154) Caesar Augustus. In: RE X 1, 1917, 478-536 hier: 516f.; R. Syme, Tacitus, Bd. 2, Oxford 1958, 517; Rohmann (Anm. 65) 127; dass das Geschichtswerk maßgeblich gewesen sei, vertreten W. Steidle, Tacitusprobleme, MH 22, 1965, 81114, hier: 105-109; Bauman (Anm. 74) 99-104; Tränkle (Anm. 76) $231 \mathrm{f}$.

${ }^{79}$ Cass. Dio 57,24,3.

${ }^{80}$ Serv. ecl. 10,1. Vgl. hierzu Speyer (Anm. 22) 59 mit Anm. 81.

${ }^{81}$ Suet. Aug. 51,3.

${ }^{82}$ Syme (Anm. 4) 141: „The collected correspondence of Cicero preserved none of the letters he received from Octavianus. That is not surprising: the editor [Atticus] knew his business. A necessary veil was cast over the earlier and private preliminaries in the anomalous alliance between oratory and arms, between the venerable consular and the revolutionary adventurer."
} 
die Publikation von Schriften, die den Princeps kompromittieren könnten, verzichtet haben. Da Atticus bereits 32 v.Chr. starb, war also schon vor der Alleinherrschaft des Augustus offensichtlich, dass man diesen besser nicht verärgerte. Zugegeben, insbesondere Atticus war aber auch Zeit seines Lebens bemüht, es sich mit keinem einflussreichen Politiker zu verscherzen, und wurde gerade deshalb kein Opfer der Bürgerkriege.

Generell war es wohl einfach, die Stimmung des Princeps vorherzusehen. Dem schon erwähnten Geschichtsschreiber Timagenes von Alexandria kündigte er die Freundschaft, als dieser sich offener Reden über Kaiser und Kaiserhaus nicht enthielt. ${ }^{83}$ Als Timagenes seine historische Schrift publizierte, verzichtete er auf die Teile, die sich mit Augustus beschäftigten. Üblicherweise wird dies so interpretiert, dass Timagenes Partien, die den Kaiser rühmten, verbrannt habe, ${ }^{84}$ es sich also um einen Racheakt handelte. Man kann dies aber auch anders deuten: Der Historiograph mag vorsichtig geworden sein und diejenigen Teile, die Octavian/Augustus betrafen, von der Publikation ausgespart haben, um einer Anklage zu entgehen. Letztlich kam Timagenes, wie bereits erwähnt, bei Asinius Pollio unter, mit dem er zuvor noch verfeindet gewesen war.

Was also kann man zusammenfassend über die Geschichtsschreibung im frühen Principat festhalten? Es gab durchaus Stimmen, die Kritik übten. Ebenso gab es Historiographen, deren Werke sich kritisch mit dem Aufstieg und der Herrschaft des ersten Kaisers auseinandersetzten. Von einer intellektuellen Opposition kann man zumindest im Falle der Historiographen aber nicht sprechen. Ein organisierter Widerstand fehlte. Titus Labienus und Cassius Severus waren verfeindet, ursprünglich auch Pollio und Timagenes. Letzterer war zudem zunächst ein Freund des Augustus gewesen. Asinius Pollio gab in frühen Jahren zwar vor, senatstreu zu sein, was ihm in der modernen Forschung den Ruf eines Republikaners eingebracht hat. Tatsächlich war er aber ebenso wie beispielsweise Plancus oder auch Messalla Corvinus ein politischer Opportunist, der den Weg des geringstmöglichen Risikos ging, eine ungewöhnlich erfolgreiche politische Karriere durchlief und - sicherlich das wichtigste Kriterium eines erfolgreichen Lebens - anders als viele seiner berühmteren Zeitgenossen im Bett starb.

Häufig genug liest man in der Forschung, dass die uns bekannten Fälle von Verfolgungen von Geschichtsschreibern in augusteischer Zeit Ausnahmen gewesen seien und dass wir von mehr Fällen wüssten, wenn es diese gegeben hätte. ${ }^{85}$ Man vergisst hierbei, dass beispielsweise Ovids Exil nirgendwo erwähnt wird außer in seinen eigenen Schriften. ${ }^{86}$ Wenn kritische Werke verbrannt worden sind - eine im augusteischen Principat erfundene Maßnahme ${ }^{87}$-, dann ist es nur natürlich, dass hiervon der Informa-

\footnotetext{
${ }^{83}$ Sen. de ira 3,23,4-6; vgl. Sen. contr. 10,5,22.

${ }^{84}$ Syme (Anm. 4) 486.

${ }^{85}$ K. A. Raaflaub/L. J. Samons II, Opposition to Augustus. In: K. A. Raaflaub/M. Toher (Hg.), Between Republic and Empire. Interpretations of Augustus and his Principate, Berkeley/Los Angeles/Oxford 1990, 417-454, hier: 445f.; Rohmann (Anm. 65) 125, 133. Siehe dagegen Rudich (Anm. 69) 7: „The evidence [...] leaves no doubt that such suppression - which included the very physical form of bookburning - repeatedly occurred under the Roman Empire, starting with the creation of the new political system, known as the Principate, by Augustus.“ $13 \mathrm{f} .:$ „One may continue to debate whether the Principate brought any social or material improvements into the Roman world, but it cannot be denied that with its arrival the very principle of intellectual exchange, be it in oral or written form, ceased to be taken for granted and became increasingly an object of abuse at the hands of the authorities."

${ }^{86}$ Rohmann (Anm. 65) 133 vermutet, dass nicht Ovids Werke hieran die Schuld trugen; anders Speyer (Anm. 22) 61f. und Rudich (Anm. 69) 19.

${ }^{87}$ Sen. contr. 10 praef. 5-8 und Suet. Cal. 16,1 mit Gelzer (Anm. 78) 516; M. R. McHugh, Historiography and Freedom of Speech: The Case of Cremutius Cordus. In: I. Sluiter/R. M. Rosen (Hg.), Free Speech in Classical Antiquity, Mnemosyne Suppl. 254, Leiden/Boston 2004, 391-408, hier: 393.
} 
tionsfluss beeinträchtigt wurde, selbst wenn einzelne Exemplare dieser Werke in Privatbeständen überlebt haben und später erneut Verbreitung fanden. ${ }^{88} \mathrm{Zu}$ beachten ist in dieser Hinsicht, dass kritische Ansichten aus Vorsicht unter Umständen gar nicht erst zu Papier gebracht wurden. So ist noch der spätere Kaiser Claudius in jungen Jahren von Mutter und Großmutter davon abgehalten worden, sich in seinem Geschichtswerk allzu detailliert mit der Zeit nach der Ermordung Caesars auseinanderzusetzen. ${ }^{89}$ Wir dürfen ferner nicht vergessen, dass die Vorgänge im römischen Reich für die Nachwelt von keiner anderen Kultur kritisch kommentiert wurden. Uns fehlt somit die Außensicht, wie sie uns bei neuzeitlichen Diktaturen vorliegt.

Überblickt man die antiken Zeugnisse insgesamt, so ist zu resümieren, dass der erste Kaiser Augustus auf kritische Stimmen seitens der Intellektuellen unterschiedlich reagierte. Wer ihm gefährlich werden konnte, musste in jedem Falle Konsequenzen fürchten. Kienasts Einschätzung, Augustus sei der selbstloseste Herrscher der Geschichte gewesen, ist somit zweifellos zu korrigieren.

\footnotetext{
${ }^{88}$ Vgl. Rohmann (Anm. 65) 128f. in Bezug auf Cremutius Cordus, dessen Werk aber in späterer Zeit offensichtlich von kritischen Passagen ,bereinigt‘ wurde; siehe Quint. inst. 10,1,104. Siehe auch Speyer (Anm. 22) 65f.; Rudich (Anm. 69) 21.

${ }^{89}$ Suet. Claud. 41,2.
} 